\title{
Very high energy observations of gamma-ray bursts with Cherenkov telescopes
}

\author{
Alessio Berti ${ }^{a, *}$ \\ ${ }^{a}$ Max Planck Institut für Physik, \\ Föhringer Ring 6, 80805 Munich, Germany \\ E-mail: aberti@mpp.mpg.de
}

Gamma-ray bursts (GRBs) are transient events releasing a large amount of energy in a short amount of time as electromagnetic radiation. In the past decades, both observational and theoretical efforts were made to understand their inner workings, both in the prompt and afterglow phase. The origin of the GeV emission detected by Fermi-LAT in several GRBs is one of the aspects of GRB physics which is currently not well understood. Observations at very high energies (VHE, E>100 GeV) by Cherenkov telescopes, given their better sensitivity, can provide crucial information to understand the mechanisms behind such high energy components. After almost 15 years of efforts, the MAGIC and H.E.S.S. collaborations finally detected their first bursts, GRB190114C and GRB180720B respectively, opening a new era in the study of GRBs. Such detections proved the presence of a new additional emission component up to TeV energies in the GRB afterglow phase, which can be explained by the synchrotron self-Compton process. Other two GRBs were also detected, GRB190829A by H.E.S.S. and GRB201216C by MAGIC, bringing more information but also revealing a complex picture to explain the origin of the VHE emission. In this context, observations by future facilities as the Cherenkov Telescope Array (CTA) observatory will play a crucial role to increase our understanding of the VHE emission in GRBs. In this contribution I will present the outstanding results accomplished in the last years by Cherenkov telescopes in the observation of GRBs and provide an overview of what can be achieved with future instruments.

\footnotetext{
*** The European Physical Society Conference on High Energy Physics (EPS-HEP2021), ***

*** 26-30 July $2021 * * *$

*** Online conference, jointly organized by Universität Hamburg and the research center DESY ***
}

\footnotetext{
${ }^{*}$ Speaker
} 


\section{Introduction}

Gamma-ray bursts are sources at cosmological distance, which the result of catastrophic events involving compact objects, such as black holes or neutron stars. As the name implies, they are detected as bright flashes of gamma-ray and hard-X radiation, with a non-thermal spectrum peaking at hundreds of $\mathrm{keV}$ and $\mathrm{MeV}$ energies. This first phase, called prompt, is characterized by high variability and a duration from a fraction of a second to hundreds of seconds. According to the duration of the prompt phase (defined by the $T_{90}$ parameter), GRBs are classified as short $\left(T_{90}<2 \mathrm{~s}\right)$ or long GRBs $\left(T_{90}>2 \mathrm{~s}\right)$. Even if this classification is based on observed quantities, it has a deeper origin in the physical systems originating GRBs. For short GRBs, it is believed that they are the result of the merger of compact objects, e.g. two neutron stars (like the GW170817/GRB 170817A event) or a neutron star and a black hole. A large fraction of long GRBs instead were associated with supernovae of Type Ic.

The prompt is followed by a second emission phase, called afterglow. The electromagnetic radiation produced in such phase is fainter, but can be detected up to days, weeks or months after the GRB onset. Moreover, the afterglow can be more easily detected at different wavelengths, from radio to $\mathrm{GeV}$ gamma rays.

One theoretical model to explain the prompt and afterglow phases is the fireball scenario, where a collimated jet is launched at relativistic velocities $(\Gamma \sim 100-1000)$ from a central engine. In the fireball scenario, the prompt emission is due to the interaction of shock waves moving at different velocities, where particles are accelerated and produce the observed prompt radiation, but also other mechanism could be at work, for example if the jet is magnetically dominated. For the afterglow, given the availability of more observational data at different wavelengths, the mechanism is more clear. As the jet moves outwards, it reaches the interstellar medium and decelerates. At the shock front (external shock) electrons are accelerated and the afterglow emission is the result of their synchrotron radiation.

Even after more than 50 years from their discovery, there are still many unresolved or debated points about GRB physics. For example, the emission process at work in the prompt phase, the composition of the jet and the particle acceleration details are still open issues. Recently, thanks to Cherenkov telescopes, it was finally proved that GRBs can emit very high energy (VHE, $E \gtrsim 100 \mathrm{GeV}$ ) gamma rays, a fact predicted in many theoretical models but without any experimental confirmation until 2019. These proceedings focus on this topic, giving an overview of the exciting results accomplished in the last three years.

\section{Observations of GRBs at VHE}

Even before the start of operations of the current generation of imaging atmospheric Cherenkov telescopes (IACTs), several models of GRBs predicted the presence of a VHE component, either in the prompt or in the afterglow, via different hadronic or leptonic mechanisms. The three major Cherenkov telescopes systems, currently operating, H.E.S.S. (Namibia), MAGIC (La Palma, Canary Islands) and VERITAS (Arizona), have the detection of GRBs in the VHE range as one of the primary scientific targets.

The task which the Cherenkov telescopes are called out for is quite challenging. Indeed, due to the 
cosmological distances of GRBs a strong absorption in the VHE range is expected even for moderate redshift, which can prevent detection. Moreover, IACTs have typically small field of views of the order of few degrees, so they need to rely on external triggers by wide field of view instrument to be able to follow-up GRBs. Finally, the duty cycle of such instruments is rather limited, given that observations can be performed only during the night, with good weather and possibly without high background due to moonlight. Therefore, the observations of some GRBs may be delayed, if not entirely canceled.

Despite these challenges, the IACTs made a huge effort in the follow-up of GRBs: MAGIC followedup around 140 GRBs since 2004, VERITAS more than 200 while H.E.S.S. observed about 60 GRBs since 2012. During the years, the follow-up strategy evolved as more information on the high energy emission was provided by Fermi-LAT. In particular, given the long lasting high energy emission in the afterglow, IACTs are now considering extended observation windows to detect a possible delayed VHE component. After many years of non-detection, finally in 2019 the MAGIC and H.E.S.S. succeeded in detecting their first GRBs at VHE, GRB 190114C and GRB 180720B respectively. This opened a new era in GRB studies, the VHE era, whose results are described in the next section.

\section{Main results from GRBs detected at VHE}

The observation of GRBs at VHE is important for different reasons. Before the detection of GRBs in the VHE range, there were many questions without answer, some of which are reported below: a) do GRBs produce VHE gamma rays? b) Is the VHE emission energetically relevant? c) What is the emission process? or do different processes contribute? d) Is there a contribution at lower energies? e) Is there VHE emission in both prompt and afterglow phases? f) Do both short and long GRBs have VHE emission? If they do, are the emission properties similar?

It is therefore clear that the contribution of Cherenkov telescopes to the GRB physics can be crucial, also in relation to other open issues. For example, the detection of VHE radiation from the prompt phase would be pivotal to finally understand the emission process at work. Also, the information provided by VHE data can help in reducing the degeneracy in GRB emission models, constraining the parameters that affect both the acceleration and radiation processes.

After almost two decades of efforts, in 2019 and 2020 the MAGIC and H.E.S.S. announced the firm detection of four GRBs in the VHE range: GRB 180720B and GRB 190829A by H.E.S.S. and GRB 190114C and GRB 201216C by MAGIC. All of these GRBs belong to the long class, but they differ in terms of energetics, distance and time of detection, as summarized in the next sections.

\subsection{GRB 180720B}

In May 2019 the H.E.S.S. collaboration announced the detection of VHE emission at the level of $5 \sigma$ from GRB 180720B. It is a long and bright GRB $\left(T_{90}=48.9 \mathrm{~s}, E_{\text {iso }}=6 \times 10^{53} \mathrm{erg}\right.$ in the 50-300 keV energy range) promptly detected by Fermi-GBM and Swift-BAT. Given that the GRB happened during the day, and according to visibility constraints, the H.E.S.S. telescopes could begin the observations only at $T_{0}+10.1 \mathrm{~h}\left(T_{0}\right.$ is the trigger time of the burst), for a total exposure of $2 \mathrm{~h}$. Before the H.E.S.S. follow-up, information about the presence of very bright high energy emission was provided by Fermi-LAT, making GRB 180720B a good candidate for possible VHE emission. Indeed, H.E.S.S. finally claimed the detection of VHE gamma rays between 100 and $440 \mathrm{GeV}$ at 


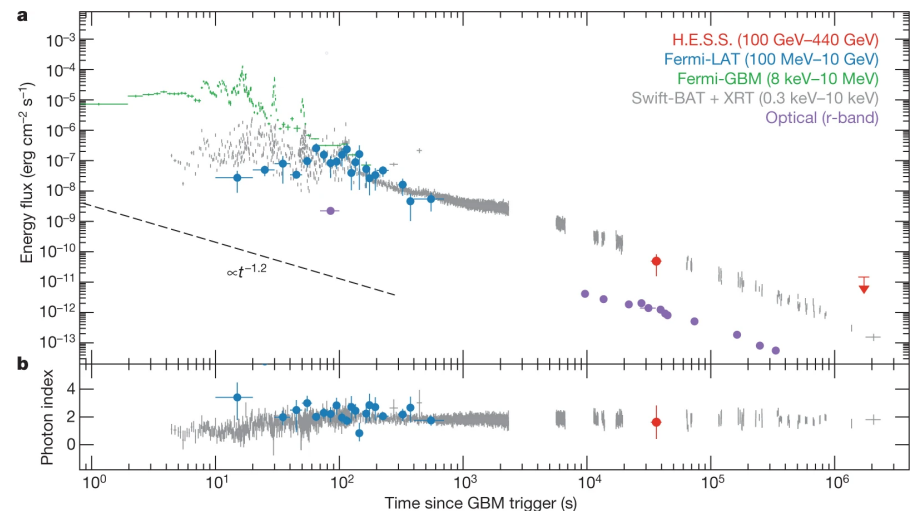

Figure 1: MWL light curve for GRB 180720B. Extracted from [1].

the $5 \sigma$ level [1]. This detection is remarkable especially because it is deep in the afterglow phase of the GRB. This proves the presence of long lasting VHE emission, similarly to what Fermi-LAT observations suggest in the HE range. Unfortunately, the photon statistics available did not allow to perform a temporal study, and the H.E.S.S. light curve has only one data point, see Fig. 1. Despite this, it can be noted that the flux level of the VHE emission is very similar to the one expected in the X-ray band at the same time, a feature that seems to be confirmed also in other GRB detection in the VHE range.

Finally, the H.E.S.S. detection suggests that the most plausible processes at the origin of the VHE gamma rays are the synchrotron emission or the synchrotron self-Compton (SSC) mechanism, with a preference for the latter. However a possible synchrotron origin is not excluded in different conditions from the ones usually considered in GRB afterglows, for example a very small coherence length of the magnetic turbulence, or different strengths of the magnetic field in the acceleration and emission zones.

\subsection{GRB 190114C}

On 14th January 2019 the MAGIC collaboration detected VHE emission from the long and bright GRB $190114 \mathrm{C}\left(T_{90}=360 \mathrm{~s}, E_{\mathrm{iso}}=3 \times 10^{53} \mathrm{erg}\right.$ in the $1-10000 \mathrm{keV}$ energy range), a burst located at $z=0.425[2,3]$. MAGIC started the follow-up at $T_{0}+57 \mathrm{~s}$ for a total exposure of $4.4 \mathrm{~h}$, detecting the source at the level of $50 \sigma$ in the first 20 minutes of observations above $300 \mathrm{GeV}$, up to $1 \mathrm{TeV}$. The MAGIC detection is remarkable because for the first time it gave the possibility to study in detail the origin of the VHE emission in a GRB, thanks also to the multi-wavelength (MWL) data collected, covering 17 orders of magnitude, from radio $(1.3 \mathrm{GHz})$ to $\mathrm{TeV}$ energies.

First of all, the emission detected by MAGIC belongs to the afterglow phase of the GRB, given the lack at lower energies of the spectral and time variability typical of the prompt phase. The VHE flux in the $0.3-1 \mathrm{TeV}$ range has a monotonic temporal decay well described by a power-law, whose index is similar to the one measured in the X-ray energy range (Swift-XRT, $1-10 \mathrm{keV}$ ), see Fig. 2. This similarity point to a process at $\mathrm{TeV}$ energies which is connected to the one at lower energies, which is the well known synchrotron radiation by relativistic electrons at the external shock in the afterglow of the GRB. However, this hypothesis is discarded: the energies of the gamma rays detected by MAGIC are well above the maximum energy of synchrotron photons, in 


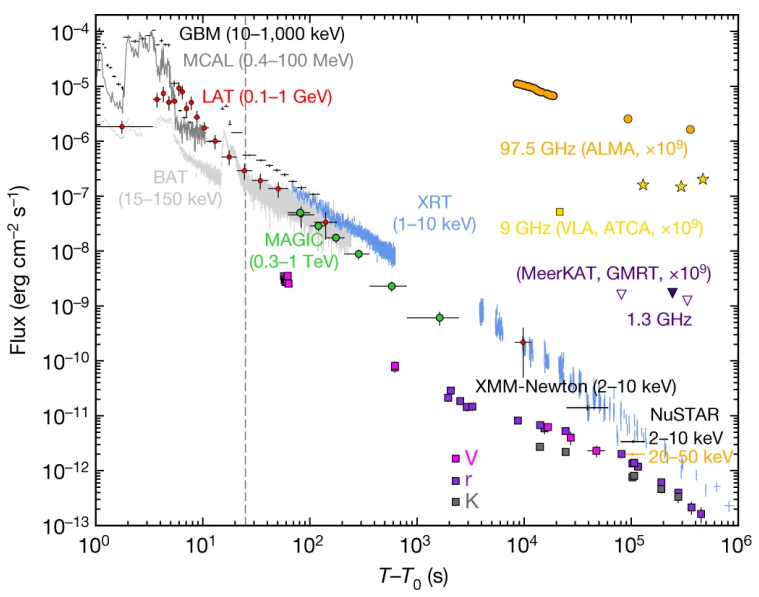

Figure 2: MWL light curve for GRB 190114C. Extracted from [3].

this specific case produced by electrons in a one zone model (burnoff limit). The MWL data in different time bins are well described by a synchrotron+SSC model, where the low energy (X-ray and HE gamma-ray range) emission is produced by synchrotron while the VHE emission is due to SSC, with internal $\gamma-\gamma$ absorption and Klein-Nishina effect included (see Fig. 3). Therefore, the MAGIC collaboration claimed the discovery of a new emission component in the afterglow of a GRB. Moreover, the parameters provided by the modeling are in agreement with the ones from previous afterglow studies, where only data up to the GeV range was used. Given that GRB 190114C does not seem to be an exceptional GRB, this may point to the possibility that VHE emission in GRBs is common and that it can be detected as soon as observing conditions are favorable.

\section{GRB 190829A}

In August 2019, the H.E.S.S. collaboration announced the detection of the long GRB 190829A [4]. This burst is quite different compared to GRB 180720B and GRB 190114C, being classified as a low luminosity GRB ( $E_{\text {iso }} \sim 2 \times 10^{50} \mathrm{erg}$ ). Additionally, it is a very close burst with $z=0.08$, one of the closest GRBs ever observed. Remarkably, the H.E.S.S. telescopes detected the GRB for three consecutive nights at the level of $21.7 \sigma, 5.5 \sigma$ and $2.4 \sigma$ respectively, with the first observation starting $4.3 \mathrm{~h}$ after the GRB onset. Also in the case of this GRB, there is a similar temporal decay of the VHE and X-ray emission. However the interpretation of the MWL data is quite different: given the low value expected for the Lorentz factor of the outflow at the time of the H.E.S.S. observation $(\Gamma<10)$, due to the Klein-Nishina effect the SSC spectrum is expected to be steeper than what obtained from VHE data. For this reason, a model where no maximum energy for the synchrotron emission is considered, so that it can extend up to the VHE range. This implies the presence of a unconventional process with high efficiency accelerating PeV electrons in magnetic fields with a strength of few Gauss, or to have a standard acceleration process with different strength for the magnetic field in the acceleration and radiation regions. This interpretation opened a debate about the mechanism driving GRB emission at VHE, arguing for example that GRB 190114C data can be modeled in the same way, at least for the second time bin. On the contrary, there have been other interpretations for GRB 190829A, considering also radio data, succeeding in modeling the 

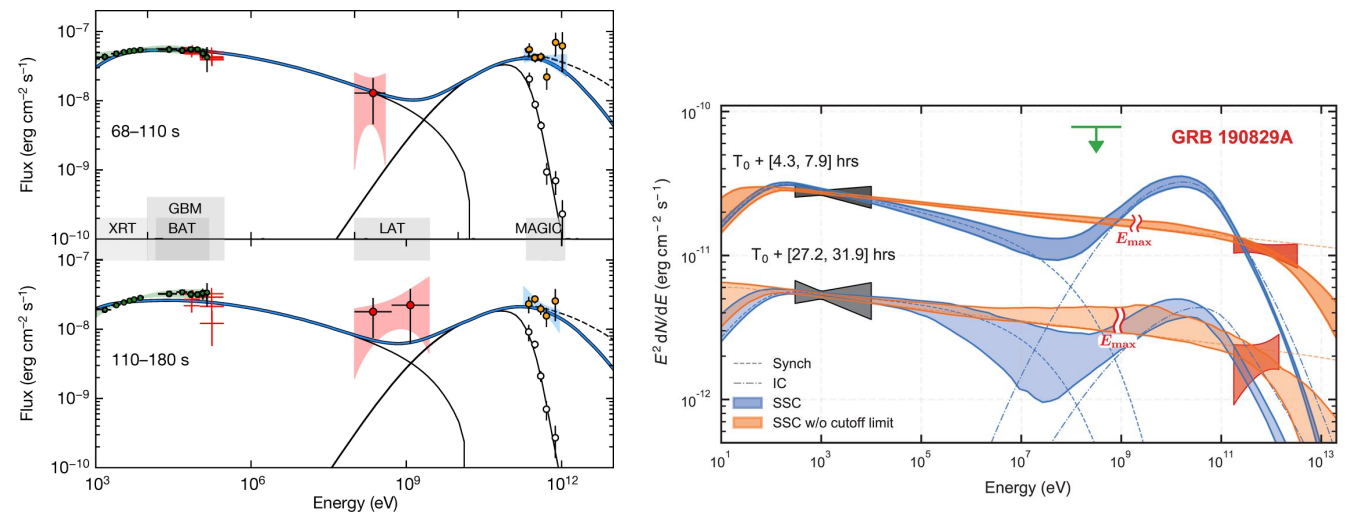

Figure 3: Spectral energy distribution and modeling of GRB 190114C (left) and GRB 190829A (right). Extracted from [3] and [4] respectively.

observations within the SSC scenario [5]. An important point is the availability of Fermi-LAT data, that can be used to constrain the presence of a dip in the spectral energy distribution (SED), as in the case of GRB 190114C, while for GRB 190829A LAT could not detect the GRB.

\section{GRB 201216C}

Finally, another long burst was detected by MAGIC on December 2020, GRB 201216C. Even if with similar energetic compared to GRB 190114C and GRB 180720B, this burst is much farther away, at $z=1.1$. MAGIC started the observation of the burst about one minute after the onset, with a detection reaching $6 \sigma$ above $\sim 100 \mathrm{GeV}$. From preliminary analyses of the MAGIC data, the photon flux monotonically decays in time, and the spectrum (both intrinsic and observed) can be described by a power law [6]. Work is ongoing on the MAGIC collaboration side to model the MWL emission, despite the lack of simultaneous data e.g. in the X-ray and high energy range (there is no LAT detection for this burst).

\section{Next challenges, prospects and conclusion}

The detection of four GRBs at VHE in the past years proved the feasibility of studying such sources with Cherenkov telescopes, both in the early and late afterglow phase. Although the four GRBs have quite some different properties, some common traits seem to emerge. Among them, the similar temporal decay and flux level of X-ray and VHE emissions stands out. The availability of other MWL data, like the one from Fermi-LAT, are crucial for the overall interpretation of the GRB emission, as exemplified by the case of GRB 190114C. The process responsible for the VHE emission is currently a heavily debated point. While SSC seems to be a good candidate, the case of GRB 190829A opened a discussion on the possible revisitation of acceleration mechanisms in GRBs (and possibly in a more general context). For sure, this uncertainty in explaining the origin of VHE emission is a clear indication that more GRBs detected in the VHE range are needed, which is surely one of the next steps in the follow-up programmes of the current IACTs.

Other challenges lie ahead for IACTs. The first is the detection of VHE emission from the prompt phase. The VHE data can give crucial information to unveil the physical process behind this emission 
phase. Furthermore, the detection of prompt VHE emission can be used, especially if the redshift of the GRB is high, to enhance the sensitivity of Lorentz Invariance Violation (LIV) searches using GRBs (e.g. see [7] for LIV limits derived using GRB 190114C data). A second challenge is the detection of VHE emission from short GRBs, an important milestone in relation to gravitational wave searches. In this context, the recent results by MAGIC on the short GRB 160821B, at $z=0.17$ [8], are encouraging. This will be crucial to understand if long and short GRBs have similar emission mechanisms. Given the intrinsic delay introduced in the follow-up by IACTs, wide field of view ground facilities like HAWC, LHAASO and SWGO may be better suited for this task.

Finally, a leap forward in GRB studies at VHE is expected to happen soon. Indeed, in the forthcoming Cherenkov Telescope Array (CTA) the Large Size Telescopes (LSTs) will have a higher sensitivity in the low energy regime, down to $\sim 20 \mathrm{GeV}$ and a higher slewing speed (any position in the sky within $20 \mathrm{~s}$ ). The first prototype, LST-1, is in its commissioning phase and is already producing interesting results on some known and transient sources [9]. The LSTs will give the opportunity to detect GRBs at high redshift and to detect dimmer events at moderate redshift.

Therefore, it is expected that in the coming years we will witness new discoveries on GRBs emission in the VHE range, fully entering in the VHE era of GRB studies.

\section{References}

[1] H. Abdalla et al., A very-high-energy component deep in the $\gamma$-ray burst afterglow, Nature 575 (2019) 464 [1911.08961].

[2] MAGIC Collaboration and L. Nava, Teraelectronvolt emission from the $\gamma$-ray burst GRB 190114C, Nature 575 (2019) 455 [2006.07249].

[3] MAGIC Collaboration et al., Observation of inverse Compton emission from a long $\gamma$-ray burst, Nature 575 (2019) 459 [2006.07251].

[4] H. E. S. S. Collaboration, P. Evans and K. Page, Revealing $x$-ray and gamma ray temporal and spectral similarities in the GRB 190829A afterglow, Science 372 (2021) 1081 [2106. 02510].

[5] O.S. Salafia, M.E. Ravasio, J. Yang, T. An, M. Orienti, G. Ghirlanda et al., Multi-wavelength view of the close-by GRB 190829A sheds light on gamma-ray burst physics, arXiv e-prints (2021) arXiv:2106.07169 [2106.07169].

[6] S. Fukami, A. Berti, S. Loporchio, Y. Suda, L. Nava, K. Noda et al., Very-high-energy gamma-ray emission from GRB 201216C detected by MAGIC, PoS ICRC2021 (2021) 788.

[7] MAGIC Collaboration and L. Nava, Bounds on Lorentz Invariance Violation from MAGIC Observation of GRB 190114C, Physical Review Letters 125 (2020) 021301 [2001.09728].

[8] V.A. Acciari et al., MAGIC Observations of the Nearby Short Gamma-Ray Burst GRB 160821B, The Astrophysical Journal 908 (2021) 90 [2012 . 07193].

[9] A. Carosi, H. Ashkar, A. Berti, P. Bordas, M. de Bony Lavergne, A. Donini et al., First follow-up of transient events with the CTA Large Size Telescope prototype, arXiv e-prints (2021) arXiv:2108.04309 [2108.04309]. 